\title{
Q31 Dissociated modulation of conditioned place-preference and mechanical hypersensitivity by a TRPA1 channel antagonist in peripheral neurpathy
}

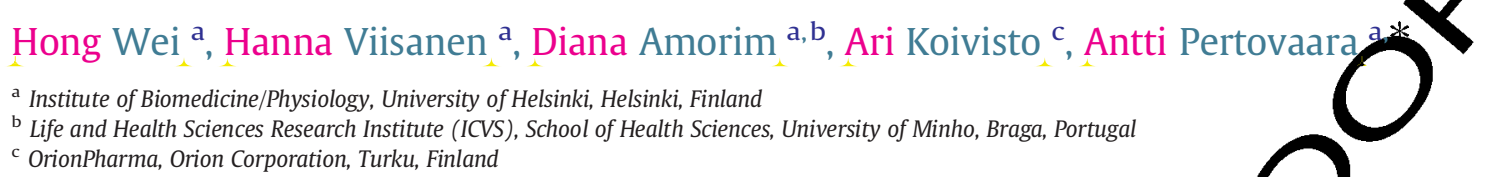

\section{A R T I C L E I N F O}

\section{Article history:}

Received 24 August 2012

Received in revised form 11 December 2012

Accepted 19 December 2012

Available online $\mathrm{xxxx}$

\section{Keywords:}

Chembridge- 5861528

Clonidine

Conditioned place-preference

Diabetic neuropathy

Mechanical hypersensitivity

Ongoing pain

Peripheral nerve injury

Transient receptor potential ankyrin 1 channel

\section{A B S T R A C T}

Transient receptor potential ankyrin 1 (TRPA1) in peripheral neuropathy, while their effect o whether blocking the TRPA1 channel induces plac-p experimental rat models of peripheral neuropathy. Diabetic neuropathy was induced by streptozotocin and spared 29 nerve injury (SNI) model of neuropatb by ation of two sciatic nerve branches. Conditioned place-preference 30 (CPP) paradigm involved pairing of druc treatment with one of the chambers of a CPP device once or four 31 times, and the time spent in each hamb was recorded after conditioning sessions to reveal place-preference. 32 The mechanical antihypersensi vit effect was assessed by the monofilament test immediately after the condi- 33 tioning sessions. Intraperitopealy $3 / \mathrm{mg} / \mathrm{kg}$; diabetic and SNI model) or intrathecally (10 $\mu \mathrm{g}$; diabetic model) 34 administered Chembridge 861528 (CHEM) was used as a selective TRPA1 channel antagonist. In diabetic and 35 SNI models of neuropathy, CF. failed to induce CPP at a dose that significantly attenuated mechanical hypersen- 36 sitivity, independent rne route of drug administration or number of successive conditioning sessions. Intrathecal 37 clonidine (an $\alpha_{2}$-ad enoce tor agonist; $10 \mu \mathrm{g}$ ), in contrast, induced CPP in SNI but not control animals. The results 38 indicate that ongoi $\quad$ bai , as revealed by CPP, is less sensitive to treatment by the TRPA1 channel antagonist than 39 mechanical hersitivity in peripheral neuropathy.

(c) 2012 Published by Elsevier Inc. 41

\section{Introduction}

Transient receptor potential ankyri calcium-permeable ion channel that of nociceptive primary afferent nerve al., 2004). In the periphery, TRPA ena of harmful stimuli to neuronal ischar ge, whereas on central endings of nociceptive nerve fibers indices glutamatergic transmission to spinal dorsal horn interneuron (see for reviews, Patapoutian et al., 2009; Stucky et al., 2009, Woran et al., 2011; Pertovaara and Koivisto, 2011; Andrade et al., 2

Peripheral neukopathi s are among pathophysiological conditions that are associated the hronic ongoing pain and hypersensitivity to cutaneous stimulation, symptoms which are significant clinical problems (Scadding and Koltzenburg, 2006). Interestingly, there is recent experimental evidence indicating that blocking the TRPA1 channel attenuates mechanical hypersensitivity induced by peripheral diabetic neuropathy (Wei et al., 2009, 2010a; Koivisto et al., 2012; Koivisto

\footnotetext{
* Corresponding author at: Institute of Biomedicine/Physiology, P.O. Box 63, University of Helsinki, 00014 Helsinki, Finland. Tel.: +358 9191 25280; fax: + 358919125302.

E-mail address: antti.pertovaara@helsinki.fi (A. Pertovaara).
}

and Pertovaara, in press) or spinal nerve injury (Eid et al., 2008; Wei 63 et al., 2011). While these findings indicate that the TRPA1 channel 64 exerts an important role in the facilitation of mechanical stimulus- 65 evoked pain in peripheral neuropathy, these findings still leave open 66 whether the TRPA1 channel is involved in maintenance of ongoing 67 neuropathic pain.

Assessment of ongoing neuropathic pain in experimental animals 69 is notoriously difficult. One approach is to apply conditioned place- 70 preference (CPP) paradigm. If animals have ongoing pain that is 71 reduced by drug treatment in one of the test chambers, the animals 72 are expected to prefer the test chamber paired with the analgesic treat- 73 ment (Sufka, 1994). Unmasking the tonic-aversive state using the CPP 74 paradigm has been successfully applied to study sustained pain in vari- 75 ous models of peripheral neuropathy (King et al., 2009; De Felice et al., 76 2011; King et al., 2011; Qu et al., 2011; He et al., 2012; Leite-Almeida et 77 al., 2012) as well as in some other experimental models of chronic pain 78 (Davoody et al., 2011; He et al., 2012; Okun et al., 2012). Here, we 79 administered a selective TRPA 1 channel antagonist in the CPP paradigm 80 that was modified from that of King et al. (2009) to study whether the 81 TRPA1 channel is involved in maintenance of ongoing neuropathic 82 pain. The experiments were performed in two models of experimental 83 peripheral neuropathy, one induced by a metabolic disorder (diabetes 84 mellitus) and one by nerve ligations (spared nerve injury, SNI). 


\section{Material and methods}

\subsection{Experimental animals}

The experiments were performed with male Hannover-Wistar rats (220-260 g; Harlan, Horst, The Netherlands) in Biomedicum Helsinki. All experiments were approved by the ethical committee for experimental animals studies of the State Provincial Office of Southern Finland (Hämeenlinna, Finland) and the experiments were performed according to the guidelines of European Communities Council Directive of 24 November 1986 (86/609/EEC). All efforts were made to minimize animal suffering, to reduce the number of animals used, and to utilize alternatives to in vivo techniques, if available. The animals were housed in polycarbonate cages with a deep layer of saw dust, one to three animals in each cage, in a thermostatically controlled room at $24.0 \pm 0.5^{\circ} \mathrm{C}$. The room was artificially illuminated from 8.30 AM to 8.30 PM. The animals received commercial pelleted rat feed (CRM-P pellets, Special Diets Services, Witham, Essex, England) and tap water ad libitum.

\subsection{Induction of diabetes mellitus}

Diabetes mellitus was induced under pentobarbitone anesthesia by tail vein injection of streptozotocin $(60 \mathrm{mg} / \mathrm{kg}$; Sigma-Aldrich, St.Louis, MO, USA) in citrate buffer ( $\mathrm{pH}$ 4.5). Streptozotocin-induced diabetes mellitus is known to cause a marked hypersensitivity to various types of stimuli (Courteix et al., 1993). While peripheral diabetic neuropathy is a complex disorder with multiple underlying mechanisms (Obrosova, 2007), the TRPA1 channel was recently shown to exert an important role in its pathophysiology (Koivisto et al., 2012). The development of diabetes mellitus was confirmed 3 and 10 days later by measurements of blood glucose concentration (One Touch Ultra, Life Scan Inc., Milpitas, CA, USA). All streptozotocin treated animals developed diabetes and had a blood glucose levero $>20 \mathrm{mmol} / \mathrm{l}$. Weight of the animals was assessed every other $\mathrm{d} y$ the animal had a weight decrease of $>20 \%$ or it showed signs ing, then the animal was immediately sacrificed by admi lethal dose of pentobarbitone.

\subsection{Techniques for producing spared nerve injury mef peripheral} neuropathy

There are a number of surgically induced odels of peripheral neuropathy (Honoré et al., 2011), of whine chose for this study the spared nerve injury (SNI) model (ecost rd and Woolf, 2000). For SNI, the unilateral axotomy and ligan the tibial and common peroneal nerves on the left side wo performed under pentobarbitone anesthesia $(60 \mathrm{mg} / \mathrm{kg}$ i.p.) as de Cribe in detail earlier (Decosterd and Woolf, 2000). Briefly, the the lateral surface of the thigh was incised and a section madectly through the biceps femoris muscle exposing the scintic erve and its three terminal branches. Following ligation and rentving 2-4 $\mathrm{mm}$ of the distal nerve stumps of the tibial and comon peroneal nerves, muscle and skin were closed in two layers. In-snam-operated animals, the surgical procedure was identical, except that the tibial and common peroneal nerves were not ligated or sectioned. After the surgery, the animals were allowed to recover before the actual testing that was performed one week after the operation.

\subsection{Surgical procedures for the installation of intrathecal catheter}

In one group of animals, drug was administered intrathecally (i.t.). For i.t. drug injections, a catheter (Intramedic PE-10, Becton Dickinson and Company, Sparks, MD) was administered into the lumbar level of the spinal cord under pentobarbital anesthesia (60 mg/kg intraperitoneally) as described in detail elsewhere (Størkson et al., 1996). Following recovery from anesthesia, the correct placing of the catheter was verified 144 by administering lidocaine (4\%, 7-10 $\mu$ followed by a $20 \mu \mathrm{l}$ of saline for 145 flushing) with a 50- $\mu$ l Hamilton syringe (Hamilton Company, Bonaduz, 146 Switzerland). Only those rats that had no motor impairment before 147 lidocaine injection but had a bilateral paralysis of hind limbs following 148 intrathecal administration of lidocaine were studied further. The installa- 149 tion of the intrathecal catheter was performed about one week before the 150 start of the actual experiments. In the actual experiments, the drugs were 151 microinjected i.t. with a 50- $\mu \mathrm{l}$ Hamilton microsyringe in a volume of $5 \mu \mathrm{l} 152$ followed by a saline flush in a volume of $20 \mu$.

\subsection{Conditioned place preference (CPP)}

154

For analysis of ongoing pain, condition pace-preference (CPP) par- 155 adigm modified from that of King et al. (20.9) was used. When using a 156 single-drug exposure paradigm, rats ntre rwent a 3 day habituation, in 157 which they were placed in autor ated PP boxes (Place Preference 158 System, San Diego Instruments, Ind Sal Diego, CA) with access to all 3159 chambers for 30 min per d du ing the first two days. The device 160 records time spent in each amb using a computer-controlled $4 \times 16 \quad 161$ array of photobeams. A thenterences between the 162 was the roughness of hoo (rough versus smooth) and the painting 163 of the walls (black tric (les versus bars on white surface). Time spent 164 in each of the bxe was recorded for $15 \mathrm{~min}$ on day 3 (D3). Rats 165 that spent mor tran $720 \mathrm{~s}$ in one of the conditioning chambers were 166 eliminated from th study. The following day (D4), all rats received a 167 morning ton of vehicle and were immediately (or in two groups, 168 15 mip after vehicle) placed in one of the pairing chambers for 169 30 min. hours later, all rats received drug (clonidine, Chembridge- 170 58 158 their combination, or in one control condition the second 171 a of vehicle) and were immediately (or in two groups, 15 min after 172 Chemrbridge-5861528) placed in the opposite chamber for $30 \mathrm{~min} . \quad 173$ On the next day (D5), $20 \mathrm{~h}$ following drug pairing, animals were 174 placed drug-free in the CPP boxes with access to all chambers. The 175 amount of time spent in each of the two chambers (saline- and 176 drug-paired) was automatically registered and used to quantify the 177 conditioning effect by drug treatment. In one test group, a multiple 178 drug exposure-paradigm was used (for details, see Section 2.7). It 179 was expected that if the animal had ongoing pain that was reduced 180 by drug treatment, the animal preferred the drug-paired chamber. $\quad 181$

\subsection{Assessment of pain-related behavior evoked by peripheral 182} test stimulation

All animals were habituated to pain testing procedures at least 1-2 h 184 per day for two days before assessing drug effects on pain behavior. Since 185 mechanical rather than heat hypersensitivity is a frequent problem 186 in patients with peripheral neuropathy (Scadding and Koltzenburg, 187 2006), the focus in testing of stimulus-evoked pain behavior was on 188 mechanically evoked responses. Hypersensitivity to cold is also com- 189 mon in peripheral neuropathies (Scadding and Koltzenburg, 2006) 190 and the spinal nerve injury-induced cold hypersensitivity has also 191 been attenuated by a TRPA1 channel antagonist (Chen et al., 2011). 192 The testing schedule in the present study, however, did not allow 193 assessing cold hypersensitivity.

To assess mechanically evoked pain behavior, the frequency of with- 195 drawal responses to the application of monofilaments (von Frey hairs) 196 to the hind paw was examined. A series of monofilaments that 197 produced forces varying from $1 \mathrm{~g}$ to $26 \mathrm{~g}$ (North Coast Medical, Inc., 198 Morgan Hill, CA) was applied in ascending order five times to the 199 plantar skin at a frequency of $0.5 \mathrm{~Hz}$. A visible lifting of the stimulated 200 hind limb was considered a withdrawal response. If the rat failed to 201 withdraw to any of the five presentations of a monofilament, the 202 response rate for the studied force level was $0 \%$. If the rat withdrew 203 every time the monofilament was applied to the paw, the response 204 rate for the studied force level was $100 \%$. Thus, an increase in the 205 
response rate represents facilitation of mechanical stimulus-evoked pain behavior (hypersensitivity). When assessing treatment effects on mechanical hypersensitivity, the treatment effect on the cumulative response rate to a series of monofilaments was calculated in the following way: the cumulative response rate after treatment - the cumulative response rate before treatment. Treatment-induced changes in cumulative response rates that were less than 0 represent treatment-induced antihypersensitivity effects. Pain behavior was assessed on day 4 of the conditioning. Testing of pain behavior was performed before placing the animal into the CPP device and immediately after its removal from the CPP device, both in the vehicle (morning) and drug (afternoon) treatment conditions.

\subsection{Course of the study}

In general, animals were tested 1-2 weeks after induction of diabetes or SNI/sham surgery. Each animal participated only in one testing condition, each of which lasted five days (see Section 2.5 for details). When single-exposure CPP paradigm was used, vehicle (morning) and drug (afternoon) were administered only on D4, whereas in the multiple-exposure CPP paradigm, vehicle and drug were administered on four consecutive days (days 1-4); in the multiple-exposure CPP paradigm the experimental procedure on days 1-4 was identical to that of day 4 in the single-exposure СРP condition. Place-preference (time spent in the vehicle- versus drug-paired chamber) was assessed on D5. Mechanical pain behavior (see Section 2.6) was assessed on D4. After completion of the study, the animals were sacrificed with a lethal dose of pentobarbitone.

\subsection{Drugs}

Chembridge-5861528, (CHEM; a derivative of HC-030031) that w synthesized by ChemBridge Corporation (San Diego, CA) was used TRPA1 channel antagonist. Its chemical structure is illustrated previous publication (Fig. 1 in Wei et al., 2009). Our calcium nagin results in human TRPA1 and TRPV1 transfected HEK that when mustard oil or 4-hydroxynonenal (4-HNE) TRPA1 channel agonist, $\mathrm{IC}_{50}$ value of CHEM was 14 14.3. 07 as a $18.7 \pm 0.3 \mu \mathrm{M}$, respectively (Wei et al., 2009). Moreo en CHEM showed no TRPA1 or TRPV1 channel agonism and no TRR 1 hannel antagonism up to a dose of $100 \mu \mathrm{M}$ (Wei et al., 2009). CWFM administered i.p. or i.t. at doses $(30 \mathrm{mg} / \mathrm{kg}$ or $10 \mu \mathrm{g}$, respect $\mathrm{el}$ have proved to have a significant mechanical antihypersensit y effect, without motor or other side-effects (e.g., Wei et al., 2009, 2010 a, 2011, 2012). With i.p. administration of CHEM, the onse of ac on is within $15 \mathrm{~min}$, the peak effect is reached at $30 \mathrm{~min}$, and theration of effect is less than two h (Wei et al., 2009). With ad inistration of CHEM, the onset of action is within $5 \mathrm{~min}$, the pe $\mathrm{k}$ eff $\mathrm{ct}$ is reached at $15 \mathrm{~min}$, and the duration of action is less tha 2 (Wei et al., 2010a). It should be noted that due to dissolvinems it was not possible to administer a higher i.t. dose of CHEM an the currently used $10 \mu \mathrm{g}$. Moreover, the currently used i.p. dose of CHEM (30 mg/ $\mathrm{kg}$ ) was the highest i.p. dose that was exped reduce selectively pathophysiological pain hypersensitivity, without a significant suppression of (physiological) nociception that is needed for protecting the tissues from damage. Clonidine, an $\alpha_{2}$-adrenoceptor agonist (Sigma-Aldrich, St.Louis, MO) was used in control experiments at an antinociceptive dose of $10 \mu \mathrm{g}$ i.t. as in the study of King et al. (2009). In general, drugs were administered immediately before placing the animal in the test chamber. However, since it may take up to 15 min before CHEM has a significant effect following i.p. administration (Wei et al., 2009) and since the lack of significant drug effect during the first 10-15 min of the pairing period of 30 min duration might prevent making the association between the i.p. CHEM treatment and the test chamber (Bardo and Bevins, 2000), in two experimental groups i.p. administration of CHEM (or vehicle) was performed 15 min before placing the animal in the test chamber.

\subsection{Statistical analysis}

When assessing CPP during the 30 min observation period on D5, 269 the absolute time each animal spent in the drug-paired chamber was 270 compared with that spent in the vehicle-paired chamber. When 271 assessing mechanical antihypersensitivity effects on D4, the CHEM- 272 induced change in the cumulative response rate to repetitive stimulation 273 with a series of monofilaments was compared with that induced by 274 vehicle treatment. These comparisons were performed using a paired 275 $t$-test. $\mathrm{P}<0.05$ was considered to represent a significant difference.

\section{Results}

3.1. CHEM- and clonidine-induced CPP 1 ntrol experiments

In healthy controls, CHEM fail to oduce CPP as revealed by equal 279 amounts of time spent in the cha (uber aired with CHEM (30 mg/kg i.p.) 280 versus vehicle $\left(t_{11}=0.11\right.$; paired with vehicle in con fol an hals, the animals spent equal amounts 282 of time in the chamber pired with the first administration of vehicle 283 as in the chamber with the second administration of vehicle 284 $\left(t_{5}=0.75\right.$; Fig. 1B $)$

In order to has a sitive control, we replicated the single-exposure 286 CPP experim it escribed by King et al. (2009) in the SNI model of 287 peripheral net (pathy. I.t. treatment with clonidine (10 $\mu$; a prototype 288 $\alpha_{2}$-adrenoceptoragonist) on D4 produced a significant CPP effect on D5 289 as reyaleo by a significantly longer time spent in the chamber paired 290 with cloniline than vehicle $\left(t_{9}=3.9, \mathrm{P}=0.0037\right.$; Fig. $\left.1 \mathrm{C}\right)$. In sham- 291 Cerate animals, i.t. treatment with clonidine on D4 failed to produce 292 a pPeffect on D5 $\left(t_{5}=0.24\right.$; Fig. 1D).

3.2. CHEM-induced CPP and mechanical antihypersensitivity effect in 294 diabetic animals

In diabetic animals, i.p. treatment with CHEM ( $30 \mathrm{mg} / \mathrm{kg}$, immediately 296 before placing the animal in the test chamber) on D4 failed to produce 297 a CPP effect on D5, as revealed by the lack of significant difference in 298 times spent in the vehicle- versus CHEM-paired chamber $\left(t_{7}=2.0 ; 299\right.$ Fig. 2A). Nor did i.t. treatment of diabetic animals with CHEM (10 $\mu \mathrm{g}) 300$ produce a significant CPP effect ( $t_{4}=0.22$; Fig. $\left.2 \mathrm{~B}\right)$.

Mechanical hypersensitivity was measured in diabetic animals by 302 assessing cumulative withdrawal response rates to repetitive stimu- 303 lation of the hind paw with a calibrated series of monofilaments be- 304 fore and after treatments. Before CHEM treatment, diabetic animals 305 were hypersensitive to mechanical stimulation as shown by an in- 306 creased mean withdrawal response rate to monofilament stimulation 307 (e.g., at the stimulus force of $8 \mathrm{~g}$ : $23 \pm 3 \%$ in diabetes versus $10 \pm 4 \%$ in 308 controls; $t_{11}=2.6, \mathrm{P}=0.023$ ). In contrast to the failure to induce a sig- 309 nificant CPP effect, CHEM produced a significant antihypersensitivity 310 effect in diabetic animals both in the i.p. $\left(t_{6}=4.9, \mathrm{P}=0.0026\right.$; Fig. $\left.2 \mathrm{C}\right) 311$ and i.t. $\left(t_{4}=3.9, \mathrm{P}=0.017\right.$; Fig. $\left.2 \mathrm{D}\right)$ treatment conditions.

\subsection{CHEM-induced CPP and mechanical antihypersensitivity effect in the 313} SNI model of neuropathy

In SNI animals, i.p. treatment with CHEM ( $30 \mathrm{mg} / \mathrm{kg}$, immediately 315 before placing the animal in the test chamber) on D4 failed to produce 316 a CPP effect on D5, as revealed by the lack of significant difference in 317 times spent in the vehicle- versus CHEM-paired chamber $\left(t_{10}=0.52 ; 318\right.$ Fig. 3A). Since pairing of the test chamber only once (on D4) with 319 CHEM failed to induce significant CPP in SNI animals, we tested whether 320 pairing the test chamber on four consecutive days (D1-D4) induced a 321 significant CPP effect on D5. However, pairing of the test chamber on 322 four consecutive days with CHEM failed to induce a significant CPP 323 effect $\left(t_{5}=0.47\right.$; Fig. 3B). 
A

Healthy controls

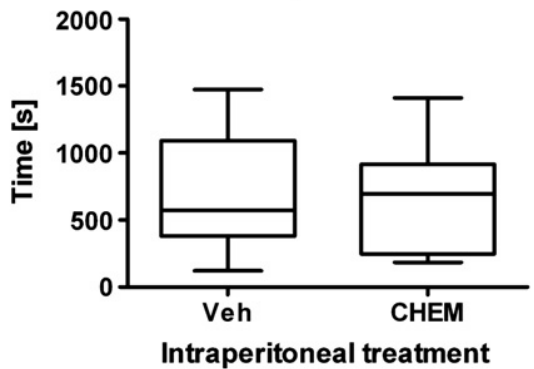

C

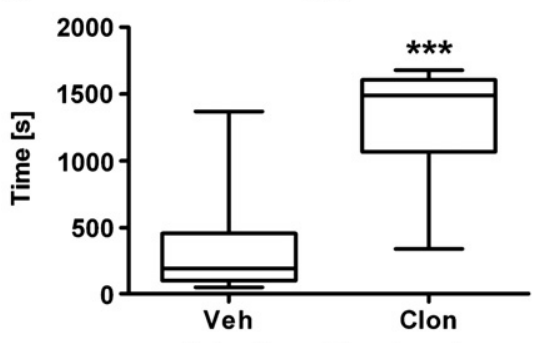

B Healthy controls

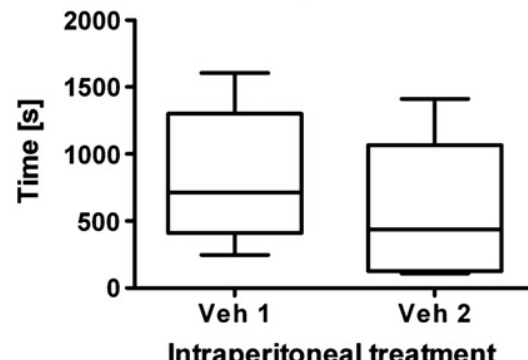

D

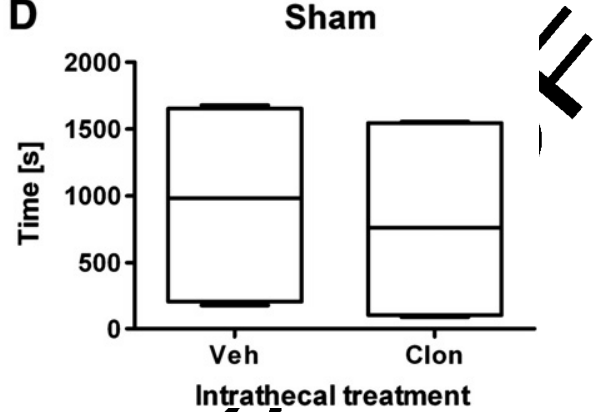

Fig. 1. Assessment of conditioned place-preference (CPP) in control experiments. (A) CPP in healthy control ans $\mathrm{s}$ ( $\mathrm{n}=12$ ) following single intraperitoneal (i.p.) treatment with Chembridge- 5861528 (CHEM, a TRPA1 channel antagonist; $30 \mathrm{mg} / \mathrm{kg}$ ). (B) CPP control experiment in which both chamsers of the CPP device were paired with vehicle in healthy controls $(n=6)$. (C) CPP in the spared nerve injury (SNI) model of peripheral neuropathy $(n=10)$ following single jumpthecal (i.t.) treatment with clonidine (Clon, an $\alpha_{2}$-adrenoceptor agonist; $10 \mu \mathrm{g})$. (D) CPP in sham-operated control animals $(n=6)$ following single i.t. treatment with clonidine ( $\mu \mathrm{g})$. . hiring of each test chamber with drug/vehicle was performed only once on day 4 and CPP was assessed as time spent in each chamber (shown by the Y-axis) on the followik day. The boxes represent median and its interquartile values, while whiskers represent the range.

Before CHEM treatments, SNI animals were hypersensitive to mechanical stimulation as shown by an increased mean withdrawa response rate to monofilament stimulation (e.g., at the stimy) force of $8 \mathrm{~g}: 50 \pm 4$ in SNI \% versus $10 \pm 4 \%$ in controls; $t_{10}=7.1,328$ 0.0001). CHEM treatment (30 mg/kg i.p.) had a significant me- 329 chanical antihypersensitivity effect assessed in SNI animals on D4 330
A

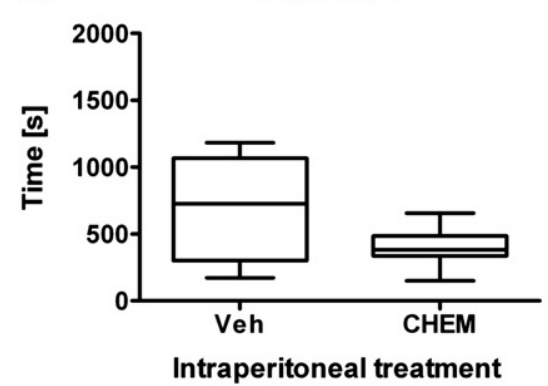

C

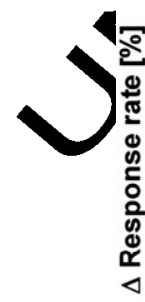

DM: hypersensitivity

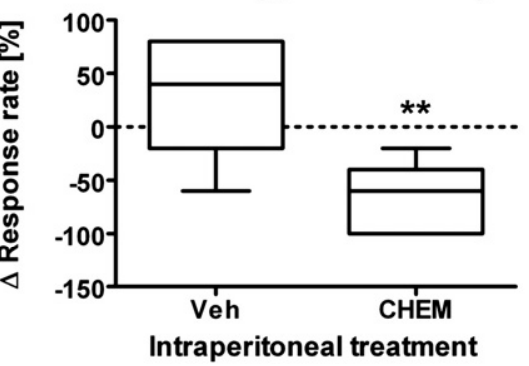

B

DM: CPP
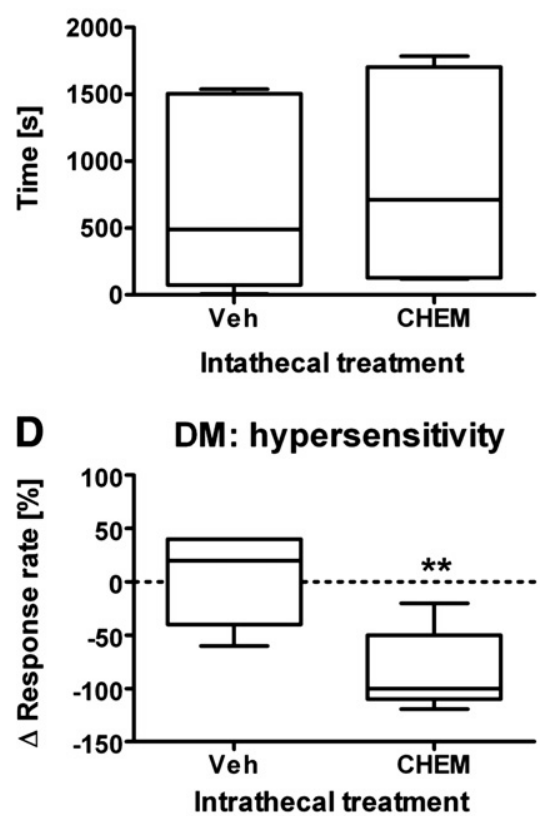

Fig. 2. Assessment of conditioned place-preference (CPP) and mechanical hypersensitivity in diabetic (DM) animals. (A) CPP and (C) the attenuation of mechanical hypersensitivity $(\mathrm{n}=7$ ) following single intraperitoneal (i.p.) treatment with Chembridge-5861528 (CHEM, a TRPA1 channel antagonist; $30 \mathrm{mg} / \mathrm{kg}$ ). (B) CPP and (D) the attenuation of mechanical hypersensitivity $(n=5)$ following single intrathecal (i.t.) treatment with CHEM $(10 \mu \mathrm{g})$. Pairing of each test chamber with drug/vehicle was performed only once on day 4 . CPP was assessed as time spent in each chamber (shown by the Y-axis) on the following day. Mechanical hypersensitivity was assessed as the cumulative response rate to a series of monofilaments. Mechanical hypersensitivity was assessed on day 4 (before and immediately after pairing one of the test chambers for 30 min with vehicle/drug administration). In graphs $C$ and $D, 0 \%$ (shown by the dotted horizontal line) represents the mean pre-drug response. Values $<0 \%$ represent a drug-induced suppression of hypersensitivity. The boxes represent median and its interquartile values, while whiskers represent the range. ${ }^{* *} \mathrm{P}<0.01$ (paired $t$-test). 
both following a single-exposure to CHEM $\left(t_{5}=4.8, \mathrm{P}=0.0047\right.$; Fig. 3C) and four exposures to CHEM ( $t_{5}=10.7, \mathrm{P}=0.0001$; Fig. 3D).

Animals in the above mentioned experiments were placed in the test chamber immediately after intraperitoneal injection of (vehicle or) CHEM, while it may take up to 15 min before i.p. administration of CHEM produces a significant antihypersensitivity effect (Wei et al., 2009). Therefore, it might be argued that the failure to induce CPP by i.p. treatment with CHEM was due to the slow onset of the significant drug effect (15 min), due to which the animals placed immediately after drug injection in the test chamber failed to associate the test chamber with the (rewarding) pain relief induced by CHEM. To exclude this possibility, a group of SNI animals were placed in the test chamber 15 min after i.p. administration of (vehicle or) CHEM. I.p. treatment with $30 \mathrm{mg} / \mathrm{kg}$ of CHEM failed to induce CPP in SNI animals $\left(t_{5}=0.3\right.$; Fig. $\left.4 \mathrm{~A}\right)$, although the animals were placed in the test chamber 15 min after i.p. drug administration (i.e., at or after the onset of the significant antihypersensitivity effect).

In case CHEM treatment abolished ongoing pain in SNI animals, it might be expected that pretreatment with CHEM prevents observing a relief of ongoing pain induced by i.t. treatment with clonidine. To address this question, we determined CPP induced by i.t. clonidine (10 $\mu \mathrm{g})$ in SNI animals that were pretreated with CHEM $(30 \mathrm{mg} / \mathrm{kg}$ i.p., 15 min prior to i.t. treatment with clonidine). In spite of i.p. pretreatment with CHEM, i.t. treatment with clonidine produced CPP in SNI animals $\left(t_{9}=2.4, \mathrm{P}=0.037\right.$; Fig. $\left.4 \mathrm{~B}\right)$.

\section{Discussion}

The main finding of this study was that the selective TRPA1 channel antagonist CHEM administered at a high systemic or intrathecal dose produced a marked mechanical antihypersensitivity effect that was not associated with CPP (an index for the drug-induced relief of ongoin pain) in experimental models of peripheral neuropathy. This finding suggests that the TRPA1 channel-mediated facilitation of sti evoked pain dissociates from mechanisms contributing to main

A
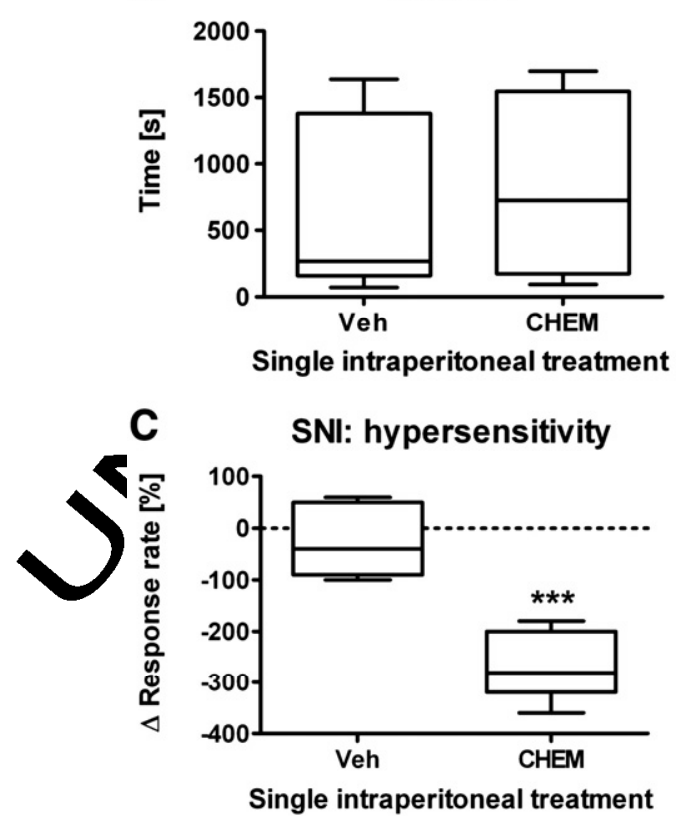

of sustained pain in peripheral neuropathy. The result allows conclud- 364 ing that ongoing pain is less sensitive to blocking the TRPA 1 channel 365 than mechanical hypersensitivity in peripheral neuropathy. It should 366 be noted that the present results don't exclude the possibility that a 367 further increase in the dose of the TRPA1 channel antagonist might 368 induce CPP in neuropathic animals. However, higher doses may not be 369 clinically feasible, due to suppression of physiological nociception that 370 helps in protecting tissues from harmful stimuli. In healthy controls, 371 the TRPA1 channel antagonist CHEM failed to induce CPP indicating 372 that the antagonist alone had neither rewarding nor aversive properties. 373

One might argue that the failure to induce CPP by administering a 374 TRPA1 channel antagonist was due to lack of ongoing pain in the 375 currently used models of peripheral neuppathy. This argument is not 376 supported by the finding that intrath calonidine produced CPP in 377 the SNI model of peripheral neuropathy o the present as in the earlier 378 study by King et al. (2009). Imporyanty intrathecal clonidine produced 379 CPP also in SNI animals that vere p etreated intraperitoneally with 380 CHEM, which finding indicates NI animals had ongoing pain that 381 was not abolished by CHE $\sim$ tro tment at a dose producing a marked 382 antihypersensitivity effe Ear er neurophysiological studies in the 383 streptozotocin-inducgen noer of diabetic neuropathy have reported 384 increased discharg ras nociceptive primary afferent nociceptive 385 nerve fibers (Khan st al., 2002) and spinal dorsal horn neurons 386 (Pertovaara a 2001; Chen and Pan, 2002). Moreover, SNI has 387 increased th ngoing discharge rate of pronociceptive medullary 388 neurons and deceased the discharge rate of antinociceptive medullary 389 neurop (tonçalves et al., 2007). These neurophysiological findings are 390 in li witl the hypothesis that diabetes or SNI induces ongoing pain. 391 O the hand, one needs to be cautious with interpretations from 392 corelative neurophysiological evidence to ongoing pain and CPP. This 393 in icated by the recent finding that systemically administered mor- 394 pline and pregabalin reduced mechanical hyperalgesia and the sponta- 395 neous discharge rate of the presumed pain-relay neurons of diabetic 396 animals, without inducing CPP (Rutten et al., 2011). Furthermore, it 397 has been pointed out that what is often considered spontaneous pain 398

B SNI: CPP

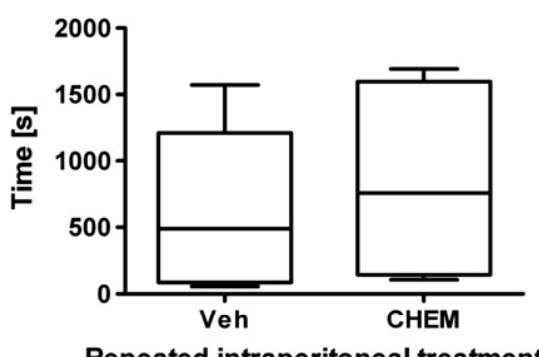

D SNI: hypersensitivity

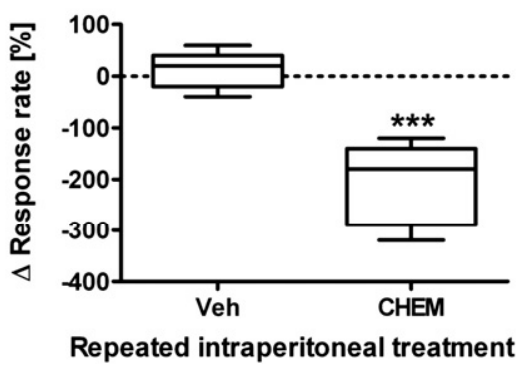

Fig. 3. Assessment of conditioned place-preference (CPP) and mechanical hypersensitivity in animals with the spared nerve injury (SNI) model of peripheral neuropathy. (A and B) CPP and ( $\mathrm{C}$ and $\mathrm{D}$ ) the attenuation of mechanical hypersensitivity following intraperitoneal (i.p.) treatment with Chembridge-5861528 (CHEM, a TRPA1 channel antagonist; 30 mg/kg). Pairing of each test chamber with drug/vehicle was performed only once on day 4 ( $A$ and $C ; n=11$ ) or on four consecutive days (B and D; $n=6$ ). CPP was assessed as time spent in each chamber (shown by the Y-axis) on the fifth day. Mechanical hypersensitivity was assessed as the cumulative response rate to a series of monofilaments. In both groups, mechanical hypersensitivity was assessed on day 4 (before and immediately after pairing one of the test chambers for 30 min with vehicle/drug administration). In graphs $\mathrm{C}$ and $\mathrm{D}, 0 \%$ (shown by the dotted horizontal line) represents the mean pre-drug response. Values $<0 \%$ represent a drug-induced suppression of hypersensitivity. The boxes represent median and its interquartile values, while whiskers represent the range. ${ }^{* * *} \mathrm{P}<0.005$ (paired $t$-test). 
A SNI: 15 min drug-chamber interval

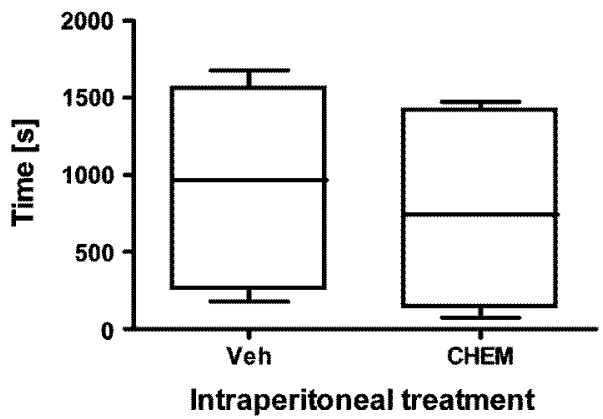

B SNI: pretreatment with CHEM

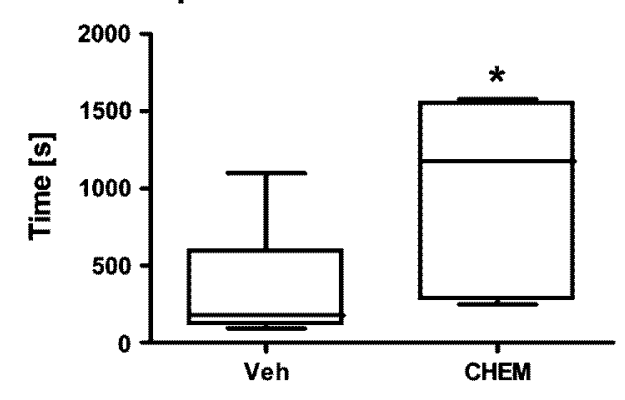

Intrathecal treatment

Fig. 4. Assessment of conditioned place-preference (CPP) in animals with the spared nerve injury (SNI) model of peripheral neuropathy. (A) CPP following single intraperitoneal treatment with vehicle (Veh) or Chembridge-5861528 (CHEM, a TRPA1 channel antagonist; $30 \mathrm{mg} / \mathrm{kg} 15 \mathrm{~min}$ prior to placing the animal in the test chamber). (B) CPP following single intrathecal treatment with vehicle or clonidine (Clon, an $\alpha_{2}$-adrenoceptor agonist; $10 \mu \mathrm{g}$ ). Animals were pretreated 15 min before intrathecal vehicle treatment with intraperitoneally administered vehicle, and $15 \mathrm{~min}$ before intrathecal clonidine treatment with intraperitoneally administered CHEM (30 mg/kg). Pairing of each test chamber with drug(s)/vehicle was performed only once on day 4. CPP was assessed as time spent in each chamber (shoy the Y-axis) on the fifth day. The boxes represent median and its interquartile while whiskers represent the range. In graph $A, n=6$ and in graph $B, n=$ (paired $t$-test). may actually represent summated pains caused by the muin of daily
life (Bennett, 2012).

The present CPP results failed to give evidepe for the TRPA1 channel in maintenance of ongo $g$ pain in peripheral neuropathy. Previous results, however, iperice that in a number of other conditions the peripheral TRPA1 hann 1 may induce afferent barrage driving ongoing pain. For examolocutaneous administration of a selective TRPA1 channel gonist in healthy control animals (e.g., Andrade et al., 2008; Tsaga(eli et al., 2010) induced sustained pain behavior. In human subjests, ectaneous administrations of a TRPA1 channel agonist (mustond or cinnamaldehyde) also produced sustained pain (Koltzenburg et al., 1992; Namer et al., 2005). Conversely, a TRPA1 chank antagonist adjacent to a wound attenuated guarding, an irkex of ongoing postoperative pain behavior in the rat (Wei et al., 201

The spinal TRPA1 channel on central terminals of nociceptive nerve fibers, in contrast, has so far been associated only with modulation of stimulus-evoked pain responses, such as secondary or central hypersensitivity (Da Costa et al., 2010; Kremeyer et al., 2010; Wei et al., 2010a, 2011; Sisignano et al., 2012; Klafke et al., 2012), or a dorsal root reflex-mediated aggravation of cutaneous neurogenic inflammation (Wei et al., 2010b), but not yet with spontaneous pain (Pertovaara and Koivisto, 2011; Wei et al., 2012). In stimulus-evoked neuropathic hypersensitivity the spinal TRPA1 channel has proved to play an important role as shown by the mechanical antihypersensitivity effect induced by spinal administration of a TRPA1 channel antagonist in nerve-injured or diabetic animals (Wei et al., 2010a, 2011).

\section{Conclusions}

426

The results of this study indicate that the TRPA1 channel-mediated 427 mechanical hypersensitivity may not reflect ongoing pain in peripheral 428 neuropathy. The significant TRPA1 channel antagonist-induced me- 429 chanical antihypersensitivity effect in SNI and diabetic animals of the 430 present study adds to the accumulating evidence indicating that selec- 431 tive TRPA1 channel antagonists are promising candidates for treating 432 pain hypersensitivity associated with peripheral neuropathy, while 433 the CPP paradigm of the present study failed to confirm their efficacy 434 against ongoing neuropathic pain.

\section{Conflict of interest}

One of the authors (A.K.) is an emplose of the pharmaceutical 437 company (OrionPharma, Finland) nat h s supported this study. 438

Acknowledgments

The study was suppored Sigrid Jusélius Foundation, Helsinki, 440 the Academy of Finlane, Hersinki, and OrionPharma, Orion Corporation, 441 Turku, Finland. Diana orim was supported by the Portuguese Science 442 Foundation (FCT/g1 nt SFRH/BD/71219/2010.

References

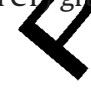

\section{Andrade L, Lui}

P. Ferreira J, Calixto JB. Pronociceptive response elicited by TRPA1 445 ation in mice. Neuroscience 2008;152:511-20. eotti FC, Calixto JB. TRPA1 antagonists as potential analgesic drugs. 447 col Ther 2012;133:189-204. Bevins RA Conditioned place preference: what does it add to our preclinical 448 tanding of drug reward. Psychopharmacology 2000;153:31-43. $\begin{array}{ll}\text { tw derstanding of drug reward. Psychopharmacology 2000;153:31-43. } & 450 \\ \end{array}$ $\begin{array}{ll}\text { mett GJ. What is spontaneous pain and who has it? J Pain 2012;13:921-9. } & 451 \\ \text { SR, Pan HL. Hypersensitivity of spinothalamic tract neurons associated with } & 452\end{array}$ diabetic neuropathy pain in rats. J Neurophysiol 2002;87:2726-33. 453

Chen J, Joshi SK, Di Domenico S, Perner RJ, Mikusa JP, Gauvin DM, et al. Selective blockade 454 of TRPA1 channel attenuates pathological pain without altering noxious cold sensation 455 or body temperature regulation. Pain 2011;152:1165-72.

Courteix C, Eschalier A, Lavarenne J. Streptozocin-induced diabetic rats: behavioural 457 evidence for a model of chronic pain. Pain 1993:53:81-8.

da Costa DS, Meotti FC, Andrade EL Leal PC Motta EM, Calixto JB. The involvement of 458 the transient receptor potential A1 (TRPA1) in the maintenance of mechanical 460 and cold hyperalgesia in persistent inflammation. Pain 2010;148:431-7.

Davoody L, Quiton RL, Lucas JM, Ji Y, Keller A, Masri R. Conditioned place preference 462 reveals tonic pain in an animal model of central pain. J Pain 2011:12:868-74. 463

De Felice M, Sanoja R, Wang R, Vera-Portocarrero L, Oyarzo J, King T, et al. Engagement 464 of descending inhibition from the rostral ventromedial medulla protects against 465 chronic neuropathic pain. Pain 2011;152:2701-9. 466

Decosterd I, Woolf CJ. Spared nerve injury: an animal model of persistent peripheral 467 neuropathic pain. Pain 2000;87:149-58. 468

Eid SR, Crown ED, Moore EL, Liang HA, Choong KC, Dima S, et al. HC-030031, a TRPA1 469 selective antagonist, attenuates inflammatory- and neuropathy-induced mechanical 470 hypersensitivity. Mol Pain 2008;4:48.

Gonçalves L. Almeida A. Pertovaara A. Pronociceptive changes in response properties of 472 rostroventromedial medullary neurons in a rat model of peripheral neuropathy. 473 Eur J Neurosci 2007;26:2188-95.

He Y, Tian X, Hu X, Porreca F, Wang ZJ. Negative reinforcement reveals non-evoked 475 ongoing pain in mice with tissue or nerve injury. J Pain 2012;13:598-607. 476

Honoré PH, Basnet A, Eljaja L, Kristensen P, Munkholm L, Andersen S. Neuropathic 477 pain models in the development of analgesic drugs. Scand J Pain 2011;2: 478 $172-7$.

Jordt SE, Bautista DM, Chuang HH, McKemy DD, Zygmunt PM, Högestätt ED, et al. 480 Mustard oil and cannabinoids excite sensory nerve fibers through the TRP channel 481 ANKTM1. Nature 2004:427:260-5.

Khan GM, Chen SR, Pan HL Ro diabetic neuropathy in rats. Neuroscience 2002;114:291-9.

King T, Vera-Portocarrero L, Gutierrez T, Vanderah TW, Dussor G, Lai J, et al. Unmasking 485 the tonic-aversive state in neuropathic pain. Nat Neurosci 2009;12:1364-6. 486

King T, Qu C, Okun A, Mercado R, Ren J, Brion T, et al. Contribution of afferent pathways to 487 nerve injury-induced spontaneous pain and evoked hypersensitivity. Pain 2011;152: 488 1997-2005.

Klafke JZ, Da Silva MA, Trevisan G, Rossato MF, Da Silva CR, Guerra GP, et al. Involvement 490 of the glutamatergic system in the nociception induced intrathecally for a TRPA1 491 gonist in rats. Neuroscience 2012;222:136-46. 492

Koivisto A, Pertovaara A. Transient receptor potential ankyrin 1 (TRPA1) ion channel in $493 \mathbf{Q 2}$ the pathophysiology of peripheral diabetic neuropathy. Scand J Pain in press. 494 http://dx.doi.org/10.1016/j.sjpain.2012.11.001. 
Koivisto A, Hukkanen M, Saarnilehto M, Chapman $\mathrm{H}$, Kuokkanen $\mathrm{K}$, Wei $\mathrm{H}$, et al. Inhibiting TRPA1 ion channel reduces loss of cutaneous nerve fiber function in diabetic animals: sustained activation of the TRPA1 channel contributes to the pathogenesis of peripheral diabetic neuropathy. Pharmacol Res 2012;65:149-58.

Koltzenburg M, Lundeberg LE, Torebjörk HE. Dynamic and static components of mechanical hyperalgesia in human hairy skin. Pain 1992;51:207-19.

Kremeyer B, Lopera F, Cox JJ, Momin A, Rugiero F, Marsh S, et al. A gain-of-function mutation in TRPA1 causes familial episodic pain syndrome. Neuron 2010;66:671-80.

Leite-Almeida H, Cerqueira JJ, Wei H, Ribeiro-Costa N, Anjos-Martins H, Sousa N, et al. Differential effects of left/right neuropathy on rats' anxiety and cognitive behavior. Pain 2012;153:2218-25.

Moran MM, McAlexander MA, Bíró T, Szallasi A. Transient receptor potential channels as therapeutic target. Nat Rev Drug Discov 2011;10:601-20.

Namer B, Seifert F, Handwerker HO, Maihöfner C. TRPA1 and TRPM8 activation in humans: effects of cinnamaldehyde and menthol. Neuroreport 2005:16:955-9.

Obrosova IG. Hyperglycemia-initiated mechanisms in diabetic neuropathy. In: Veves A, Malik RA, editors. Diabetic neuropathy: clinical management. Totowa, NJ: Humana Press; 2007. p. 69-90.

Okun A, Liu P, Davis P, Ren J, Remeniuk B, Brion T, et al. Afferent drive elicits ongoing pain in a model of advanced osteoarthritis. Pain 2012;153:924-33.

Patapoutian A, Tate S, Woolf CJ. Transient receptor potential channels: targeting pain at the source. Nat Rev Drug Discov 2009;8:55-68.

Pertovaara A, Koivisto A. TRPA1 ion channel in the spinal dorsal horn as a therapeutic target in central pain hypersensitivity and cutaneous neurogenic inflammation. Eur J Pharmacol 2011;666:1-4.

Pertovaara A, Wei H, Kalmari J, Ruotsalainen M. Pain behavior and response properties of spinal dorsal horn neurons following experimental diabetic neuropathy in the rat: modulation by nitecapone, a COMT inhibitor with antioxidant properties. Exp Neurol 2001;167:425-34.

Qu C, King T, Okun A, Lai J, Fields HL, Porreca F. Lesion of the rostral anterior cingulate cortex eliminates the aversiveness of spontaneous neuropathic pain following partial or complete axotomy. Pain 2011;152:1641-8.

Rutten K, Tzschentke TM, Christoph J, De Vry J, Schröder W. Critical appraisal of the conditioned place preference paradigm as readout for spontaneous pain in streptozotocin-induced diabetic rats. Eur J Pain Suppl 2011;5:220.
Scadding JW, Koltzenburg M. Painful peripheral neuropathies. In: McMahon SB, 531 Koltzenburg M, editors. Wall and Melzack's textbook of pain. 5th edition. China: 532 Elsevier; 2006. p. 973-99.

Sisignano M, Park CK, Angioni C, Zhang DD, von Hehn C, Cobos EJ, et al. 5.6-EET is 534 released upon neuronal activity and induces mechanical pain hypersensitivity via 535 TRPA1 on central afferent terminals. J Neurosci 2012;32:6364-72. 536

Størkson RV, Kjørsvik A, Tjølsen A, Hole K. Lumbar catheterization of the spinal 537 subarachnoid space in the rat. J Neurosci Methods 1996;65:167-72.

Story GM, Peier AM, Reeve AJ, Eid SR, Mosbacher J, Hricik TR, et al. ANKTM1, a TRP-like 539 channel expressed in nociceptive neurons, is activated by cold temperatures. Cell 540 2003;112:819-29.

Stucky CL, Dubin AE, Jeske NA, Malin SA, McKemy DD, Story GM. Roles of transient 542 receptor channels in pain. Brain Res Rev 2009;60:2-23. 543

Sufka KJ. Conditioned place preference paradigm: a novel approach for analgesic drug 544 assessment against chronic pain. Pain 1994;58:355-66.

Tsagareli MG, Tsiklauri N, Zanotto KL, Carstens MI, Klein AH, Sawyer CM, et al. Behavioral 546 evidence of thermal hyperalgesia and mechanipal allodynia induced by intradermal 547 cinnamaldehyde in rats. Neurosci Lett 2010;4 3:233-6. 548

Wei H, Hämäläinen MM, Saarnilehto M, Ke As A, Pertovaara A. Attenuation of 549 mechanical hypersensitivity by an antagonis of the TRPA1 ion channel in diabetic 550 animals. Anesthesiology 2009;111:1 551

Wei H, Chapman H, Saarnilehto M, Kuok anen K, doivisto A, Pertovaara A. Roles of cutane- 552 ous versus spinal TRPA1 channels (mech nical hypersensitivity in the diabetic or 553 mustard oil-treated non-diabetic veruropharmacology 2010a;58:578-84. 554

Wei H, Koivisto A, Pertovaara Spina TRPA1 ion channels contribute to cutaneous 555 neurogenic inflammation (the ra Neurosci Lett 2010b;479:253-6. 556

Wei H, Koivisto A, Saarnilehto M Chaman H, Kuokkanen K, Hao B, et al. Spinal transient 557 receptor potential an 1 channel contributes to central pain hypersensitivity in 558 various pathophysi ogil conditions in the rat. Pain 2011:152:582-91. 559

Wei H, Karimaa M, Kol, oo T, Koivisto A, Pertovaara A. Transient receptor potential 560

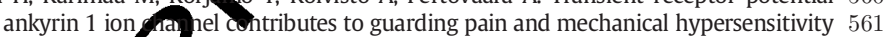
$\begin{array}{ll}\text { in a rat mod of ostoperative pain. Anesthesiology 2012;117:137-48. } & 562 \\ & 563\end{array}$

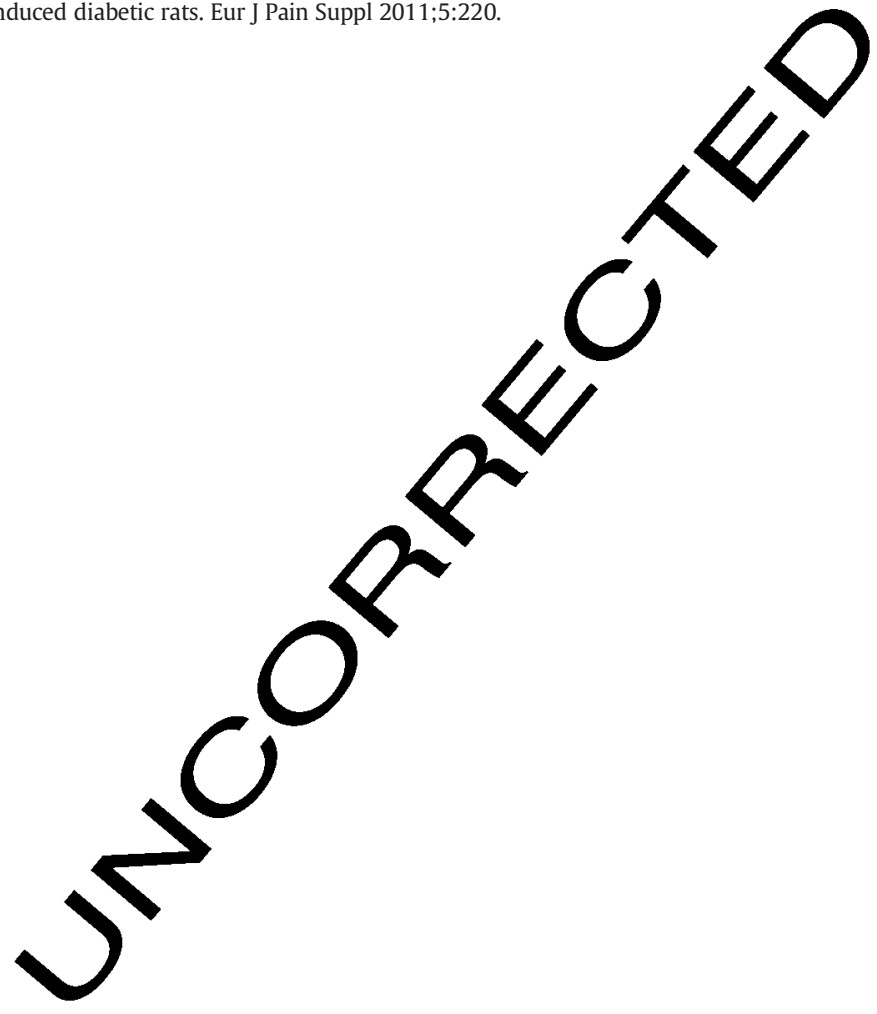

